\title{
Mu Opioid Receptor Modulation of Dopamine Neurons in the Periaqueductal Gray/Dorsal Raphe: A Role in Regulation of Pain
}

\author{
Chia $\mathrm{Li}^{1,2,5}$, Jonathan A Sugam ${ }^{2,3,5}$, Emily G Lowery-Gionta ${ }^{2,3}$, Zoe A McElligott ${ }^{2,4}$, Nora M McCall ${ }^{2,3}$, \\ Alberto J Lopez ${ }^{2,3}$, Jessica M McKlveen ${ }^{2,3}$, Kristen E Pleil ${ }^{2,3}$ and Thomas L Kash ${ }^{*, 2,3}$ \\ 'Neurobiology Curriculum, University of North Carolina at Chapel Hill, Chapel Hill, NC, USA; ${ }^{2}$ Bowles Center for Alcohol Studies, University of \\ North Carolina at Chapel Hill, Chapel Hill, NC, USA; ${ }^{3}$ Department of Pharmacology, University of North Carolina at Chapel Hill, Chapel Hill, \\ NC, USA; ${ }^{4}$ Department of Psychiatry, University of North Carolina at Chapel Hill, Chapel Hill, NC, USA
}

\begin{abstract}
The periaqueductal gray (PAG) is a brain region involved in nociception modulation, and an important relay center for the descending nociceptive pathway through the rostral ventral lateral medulla. Given the dense expression of mu opioid receptors and the role of dopamine in pain, the recently characterized dopamine neurons in the ventral PAG (VPAG)/dorsal raphe (DR) region are a potentially critical site for the antinociceptive actions of opioids. The objectives of this study were to (I) evaluate synaptic modulation of the vPAG/ DR dopamine neurons by mu opioid receptors and to (2) dissect the anatomy and neurochemistry of these neurons, in order to assess the downstream loci and functions of their activation. Using a mouse line that expresses eGFP under control of the tyrosine hydroxylase (TH) promoter, we found that mu opioid receptor activation led to a decrease in inhibitory inputs onto the vPAG/DR dopamine neurons. Furthermore, combining immunohistochemistry, optogenetics, electrophysiology, and fast-scan cyclic voltammetry in a TH-cre mouse line, we demonstrated that these neurons also express the vesicular glutamate type 2 transporter and co-release dopamine and glutamate in a major downstream projection structure - the bed nucleus of the stria terminalis. Finally, activation of TH-positive neurons in the vPAG/DR using Gq designer receptors exclusively activated by designer drugs displayed a supraspinal, but not spinal, antinociceptive effect. These results indicate that VPAG/DR dopamine neurons likely play a key role in opiate antinociception, potentially via the activation of downstream structures through dopamine and glutamate release.

Neuropsychopharmacology (2016) 4I, 2122-2132; doi:I0.1038/npp.2016.12; published online 27 April 2016
\end{abstract}

\section{INTRODUCTION}

Dopamine (DA) signaling has been demonstrated to play an important role in the modulation of pain. Diseases impacted by DA dysfunction, such as Parkinson's disease, addiction, and depression, are often comorbid with symptoms of chronic pain (Weaver and Schnoll, 2002; Beiske et al, 2009; Tykocki et al, 2013; Heimer et al, 2015; Hong et al, 2015). Animal studies have implicated that DA modulates nociception in various regions of the central nervous system, including the basal ganglia, thalamus, insular cortex, anterior cingulate cortex, and periaqueductal gray (PAG) (Wood, 2008; Jarcho et al, 2012). The PAG is of particular interest, as it contains a relatively understudied population of DA neurons and has been shown to play a role in opiate-

*Correspondence: Dr TL Kash, Department of Pharmacology, Bowles Center for Alcohol Studies, CB 7I78, University of North Carolina at Chapel Hill, 104 Manning Drive, Chapel Hill, NC 27599, USA,

Tel: + | 9198437867, Fax: + | 9199665640, E-mail: tkash@email.unc.edu

${ }^{5}$ These two authors contributed equally to this work.

Received 22 April 2015; revised 8 January 2016; accepted 8 January 2016; accepted article preview online 21 January 2016 mediated antinociception, likely due to its dense expression of mu opioid receptors (MORs). Moreover, antinociceptive effects have been demonstrated via electrical stimulation of the PAG (Mayer et al, 1971), local opioid (Akaike et al, 1978), cannabinoid (Finn et al, 2003), and GABA antagonism within the PAG (Budai and Fields, 1998). While recent studies have investigated the electrophysiological properties of the DA neuron population in the ventral PAG/dorsal raphe (vPAG/DR) (Dougalis et al, 2012; Li et al, 2013), little is known regarding their modulation by opioid receptors.

Chemical lesion of PAG DA neurons attenuates opioid induced antinociception, suggesting that this neuronal population is a critical site of action (Flores et al, 2004). Activation of opioid-class receptors in mice presynaptically inhibits GABAergic transmission in the ventral tegmental area (VTA), a DA-rich brain region, resulting in the disinhibition of the DA neurons, and an increase in their activity (Tan et al, 2010; Xie et al, 2012). Similar to the VTA, MOR activation in the PAG reduces presynaptic GABAergic neurotransmission in rats (Vaughan et al, 1997; Hahm et al, 2011; Lecca et al, 2012). These studies suggest opioid-mediated regulation of GABAergic tone modulates 
the activity of vPAG/DR DA neurons and antinociception. There has been no direct evidence showing opioid-mediated changes in inhibitory transmission in vPAG/DR DA neurons, however, nor has there been any examination of downstream synaptic or functional consequences of the activation of vPAG/DR DA neurons.

In recent years, mounting evidence has suggested that midbrain DA neurons in the VTA and substantia nigra can co-release neurotransmitters such as GABA (Tritsch et al, 2012) or glutamate (Hnasko et al, 2010; Stuber et al, 2010; Tecuapetla et al, 2010). It has yet to be determined if activation of vPAG/DR DA neurons leads solely to the release of DA or also to the co-release of other neurotransmitters. In this study, we used optogenetics in vPAG/ DR TH neurons to examine neurotransmitter release in the bed nucleus of the stria terminalis (BNST), a projection target of vPAG/DR. This allowed us to further clarify the role of these neurons in downstream neural modulation. Finally, we directly assessed the role of the vPAG/DR DA neurons in regulation of pain-related behaviors using a chemogenetic approach. Our results support the hypothesis that vPAG/DR DA neurons are a critical cellular site of action of the antinociceptive actions of opiates.

\section{MATERIALS AND METHODS}

\section{Animals and Husbandry}

Adult male tyrosine hydroxylase-eGFP (TH-eGFP) mice on a Swiss Webster background, or TH-cre on a C57BL/6J background (aged between 5 and 9 weeks, age matched for behavioral assays), were obtained from the Mutant Mouse Regional Resource Centers supported by NIH, bred and used inhouse in accordance with an animal use protocol approved by the University of North Carolina-Chapel Hill (IACUC). GAD67 mice used for TH immunohistochemistry were obtained from Dr Ben Philpot at the University of North Carolina-Chapel Hill. Co-localization of vesicular glutamate type 2 transporter (vGlut2) and TH utilized a mouse progeny bred inhouse via a cross between a vGlut2-cre mouse obtained from Dr Brad Lowell at the Beth Israel Deaconess Medical Center at Harvard University Medical School and an Ai3 mouse obtained from Dr William Snider at the University of North Carolina-Chapel Hill. Mice were group-housed under a 12-12 h light cycle, with lights on at 0700 hours daily. Mice were given ad libitum access to rodent chow and water.

\section{Stereotaxic Surgery}

Mice were anesthetized with isoflurane and placed in a stereotaxic frame (Kopf Instruments, Tujunga, CA). A microinjection needle (32-gauge) was connected to a $1 \mu \mathrm{l}$ Hamilton syringe mounted onto a digital micro-infusion pump (KD Scientific, Holliston, MA). For electrophysiological and optogenetic experiments, TH-cre mice were injected with an AAV5-Efl $\alpha$ vector encoding DIO-Ch2 (H134R)-mCherry, unilaterally at an angle of $20^{\circ}$ into the vPAG/DR (250 nl, coordinates from Bregma AP - 4.5, ML $0.0, \mathrm{DV}-2.8)$. After a postsurgical period of 6 weeks, the mice were killed and prepared for slice electrophysiology or voltammetry. For behavioral experiments, TH-cre mice were injected with an AAV8-Efl $\alpha$ vector encoding DIO-hM3DqmCherry or the control fluorophore (AAV8-Efl $\alpha$ vector encoding mCherry) at a $20^{\circ}$ angle $(500 \mathrm{nl}, \mathrm{AP}-4.5, \mathrm{ML} \mathrm{0.0}$, DV -3.1). For vPAG/DR and VTA DA terminal tracing experiments, TH-Cre mice were co-injected with AAV5-EF1 $\alpha$-DIO-enhanced yellow fluorescent protein (eYFP) in the vPAG/DR at a $20^{\circ}$ angle ( $400 \mathrm{nl}$, AP $-3.7, \mathrm{ML}$ $0.0, \mathrm{DV}-3.2)$ and AAV5-EF1 $\alpha$-DIO-mCherry in the VTA (coordinate from Bregma: AP - 3.2, ML - 0.4, DV - 4.8). Native fluorescence was imaged 4 weeks post-surgery.

\section{Electrophysiology Brain Slice Preparation}

Mice were decapitated under isoflurane anesthesia and their brains were rapidly removed and placed in ice-cold sucrose artificial cerebrospinal fluid (ACSF): (in mM) 194 sucrose, 20 $\mathrm{NaCl}, 4.4 \mathrm{KCl}, 2 \mathrm{CaCl}_{2}, 1 \mathrm{MgCl}_{2}, 1.2 \mathrm{NaH}_{2} \mathrm{PO}_{4}, 10.0$ glucose, and $26.0 \mathrm{NaHCO}_{3}$ saturated with $95 \% \mathrm{O}_{2} / 5 \% \mathrm{CO}_{2}$. Three hundred micron slices were prepared using a Leica VT1200 vibratome (Wetzlar, Germany).

\section{Slice Whole-Cell Electrophysiology}

Brain slices containing the PAG were obtained and stored at approximately $30^{\circ} \mathrm{C}$ in a heated, oxygenated holding chamber containing ACSF (in mmol/l) $124 \mathrm{NaCl}, 4.4 \mathrm{KCl}, 2$ $\mathrm{CaCl}_{2}, 1.2 \mathrm{MgSO}_{4}, 1 \mathrm{NaH}_{2} \mathrm{PO}_{4}, 10.0$ glucose, and 26.0 sodium bicarbonate before being transferred to a submerged recording chamber maintained at approximately $30^{\circ} \mathrm{C}$ (Warner Instruments, Hamden, Connecticut). Recording electrodes (3-5 M $\Omega$ ) were pulled with a Flaming-Brown Micropipette Puller (Sutter Instruments, Novato, CA) using thin-walled borosilicate glass capillaries.

For $\mathrm{mu}$ opioid (MOR) inhibitory neurotransmission experiments, recording electrodes were filled with (in $\mathrm{mmol} / \mathrm{l}) 130 \mathrm{CsCl}, 10$ 4-(2-hydroxyethyl)-1-piperazineethanesulfonic acid, 2 QX-314, 10 EGTA, 2 ATP, 0.2 GTP, $\mathrm{pH}$ 7.4, 290-295 mOsmol. Experiments were conducted under voltage clamp configuration and cells were held at $-70 \mathrm{mV}$. Inhibitory postsynaptic currents (IPSCs) were pharmacologically isolated with $3 \mathrm{mmol} / \mathrm{L}$ kynurenic acid to block $\alpha$-amino-3-hydroxy-5-methyl-4-isoxazole-propionic acid and $N$-methyl-D-aspartate receptor-dependent postsynaptic currents. To isolate miniature IPSCs (mIPSCs), tetrodotoxin (TTX, $0.5 \mu \mathrm{mol} / \mathrm{l}$ ) was added to the perfusing ACSF solutions described above. For evoked IPSC experiments, currents were induced via twisted bipolar nichrome wire placed lateral to the recording electrode. Electric output was set to stimulate at $0.1 \mathrm{~Hz}$ paired pulses, between 5 and $50 \mathrm{~V}$ with a $100-150 \mu$ s duration.

Signals were acquired via a Multiclamp 700B amplifier (Molecular Devices, Sunnyvale, California), digitized at $20 \mathrm{kHz}$, filtered at $3 \mathrm{kHz}$, and analyzed using Clampfit 10.2 software (Molecular Devices). Input resistance and access resistance were continuously monitored during experiments. Experiments in which changes in access resistance were greater than $20 \%$ were not included in the data analysis.

\section{Fast-Scan Cyclic Voltammetry}

BNST slices were prepared from TH-cre ${ }^{(+/-)}::$ChR2 mice for fast-scan cyclic voltammetry (FSCV) experiments as stated 
above for electrophysiology and perfused with ACSF. Carbon fiber microelectrodes (CFMEs) were made as previously described (Bath et al, 2000) and cut to $75 \mu \mathrm{m}$. For DA detection, the CFME was positioned into the dorsal-lateral (dl) BNST. A potential of $-0.4 \mathrm{~V}(v s \mathrm{Ag} / \mathrm{AgCl})$ was applied to the CFME and then rapidly ramped to $1.3 \mathrm{~V}$ (at $400 \mathrm{v} / \mathrm{s}$ ) at a rate of $10 \mathrm{~Hz}$ (Tar Heel CV software, Labview). Following $2 \mathrm{~s}$ of background scans, the slices were stimulated with light pulses from a blue LED (Thorlabs, $437 \mathrm{~nm}, 5 \mathrm{~ms}$ pulse width, $1 \mathrm{~mW}$ ) via a $\times 40$ objective. Following detection, background subtracted cyclic voltammograms (CVs) were analyzed using HDCV software (Labview). Oxidative currents were detected at the peak of oxidative potential corresponding to $0.6 \mathrm{~V}$ on the CV.

\section{Behavioral Experiments}

Behavioral assays were conducted at least 3 weeks following stereotaxic $\mathrm{Gq}$ designer receptors exclusively activated by designer drug (DREADD) viral injection. Animals were aged between 5 and 9 weeks at the time of stereotaxic surgery. Littermate controls were age matched with Gq DREADD animals to ensure that differences in behavioral phenotypes were not the result of differences in age. On the day of behavioral assays, $1 \mathrm{mg} / \mathrm{kg} \mathrm{CNO}$ was administered to each animal via intraperitoneal (i.p.) injection $45 \mathrm{~min}$ before its respective trial to ensure Gq DREADD activation during the assay.

\section{Open Field}

Each animal was placed in the center of an open field chamber measuring $25(\mathrm{~L}) \times 25(\mathrm{~W}) \times 25 \mathrm{~cm}(\mathrm{H})$ for a 30 -min session. The chamber was enclosed in a fan-ventilated soundattenuation chamber with interior white LED illumination at 15 lux. Locomotor activity was measured by distance traveled via the tracking software Noldus Ethovision 10.0.

\section{Hot Plate and Tail Flick Assay}

Both assays investigated the latency of response to heat-induced pain. In the hot plate assay, each mouse was gently placed into a cylindrical enclosure onto the heated platform maintained at $52.5^{\circ} \mathrm{C}$. Each trial lasted $60 \mathrm{~s}$ to minimize tissue damage. The first occurrence of hindpaw licking, withdrawal, or jumping was recorded as response to heat-induced pain (Espejo et al, 1994). In the tail flick assay, each mouse was placed in a restraint tube and their tail placed over the test apparatus approximately $2-3 \mathrm{~cm}$ from the end. The latency to remove the tail from the apparatus was measured. The intensity of the heated lamp stimulus was set such that the baseline latency for tail removal was 3-5 s. For each time point, two readings were taken $1 \mathrm{~cm}$ apart on the tail and averaged together. Animals were tested prior to $\mathrm{CNO}$ injection, $30 \mathrm{~min}$ following injection, and $1 \mathrm{~h}$ following injection. Each trial lasted no more than $10 \mathrm{~s}$ to maximize behavioral responses and minimize tissue damage. During the first day of testing, animals were placed into the restraint tube, without any habituation to the apparatus, and tested immediately on the tail flick. To ensure that stress associated with the restraint tube did not block any analgesic effects of Gq DREADD activation, animals underwent two more training sessions in which saline injections were administered prior to animals being placed in the restraint tube and analgesia was tested. The following day, animals underwent test sessions with $\mathrm{CNO}$ injections prior to analgesia testing (no differences to initial test, data not shown).

\section{Immunohistochemistry}

Mice were anesthetized with Avertin and perfused transcardially with chilled $0.01 \mathrm{M}$ phosphate-buffered saline (PBS), immediately followed by $4 \%$ paraformaldehyde PBS. Brains were extracted and post-fixed in $4 \%$ paraformaldehyde for $24 \mathrm{~h}$ and then immersed in a 30\% sucrose solution for $48 \mathrm{~h}$, both at $4{ }^{\circ} \mathrm{C}$. Coronal sections $45 \mathrm{um}$ in thickness were collected using a Leica VT1000S vibratome (Leica Microsystems, Nussloch, Germany) and stored in a 50\% glycerol solution at $-20^{\circ} \mathrm{C}$ until immunohistochemistry was performed. Slices were rinsed four times for $5 \mathrm{~min}$ in chilled PBS, followed by a 30-min incubation in 50\% methanol, a 5-min incubation in 3\% hydrogen peroxide, three 10-min PBS washes, a $30-\mathrm{min}$ incubation in $0.5 \%$ Triton X-100 in PBS solution, and a 10-min PBS wash. After a final 1-h incubation in a blocking solution made of $0.1 \%$ Triton X-100/10\% Normal Donkey Serum in PBS, the tissue slices were incubated overnight $(\sim 18 \mathrm{~h})$ at room temperature with their respective primary antibodies diluted in $0.1 \%$ Triton X-100/10\% Normal Donkey Serum in PBS blocking solution (anti-TH (1:1000), Immunostar 22941-907001; anti-Green Fluorescent Protein (1:500), Aves Laboratories, GFP-1020). Slices were rinsed three times for $10 \mathrm{~min}$ in chilled PBS before incubating for $2 \mathrm{~h}$ in their respective secondary antibodies diluted in PBS (Alexa Fluor 647 Donkey anti-Mouse $(1: 200)$, TH; Alexa Fluor 488 Donkey anti-Chicken $(1: 200)$, GFP; Jackson Immuno Research). Tissue was rinsed four times for $10 \mathrm{~min}$ in chilled PBS, and mounted using Vecta-Shield Mounting Medium (Vector Laboratories, Burlingame, CA, $\mathrm{H}-1000)$ prior to image collection.

\section{Statistics}

Effects of drugs during electrophysiological recordings were evaluated by comparing the magnitude of the dependent measure (mIPSC frequency and amplitude) between the baseline, wash-on (when drug had reached maximal effect at $10 \mathrm{~min}$ ), and antogonist periods using one-way ANOVA repeated measure test. The effects of antagonists/blockers on the ability of drugs to modulate synaptic transmission were compared using $t$-tests during the washout period. All values given for drug effects throughout the article are presented as mean \pm SEM.

\section{Drugs}

Tetrodotoxin citrate (TTX, $500 \mathrm{nM}$ ) and kynurenic acid $(3 \mathrm{mM})$ were obtained from Abcam and dissolved in water. Clozapine-n-oxide (CNO, 10uM for slice electrophysiology bath application, $1 \mathrm{mg} / \mathrm{kg}$ for behavioral testing) stock was dissolved in DMSO and diluted with ACSF (electrophysiol-

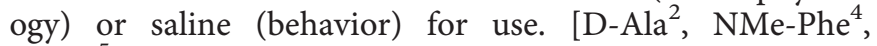
Gly-ol ${ }^{5}$-enkephalin (DAMGO) (300 nM) and D-Phe-CysTyr-D-Trp-Orn-Thr-Pen-Thr-NH $\mathrm{NH}_{2}$ (CTOP) $(1 \mu \mathrm{M})$ were purchased from Tocris and dissolved in water. 


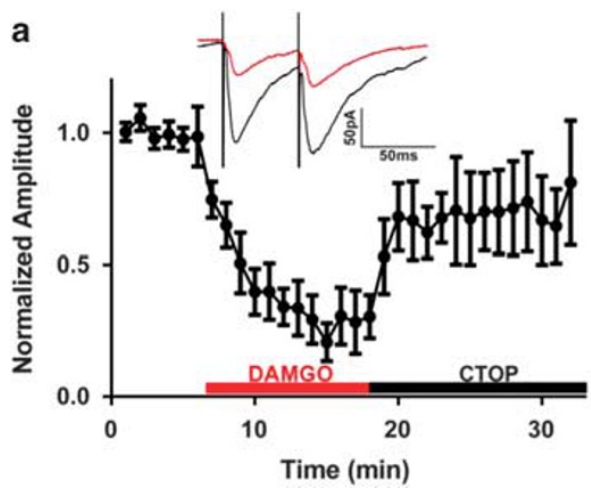

b

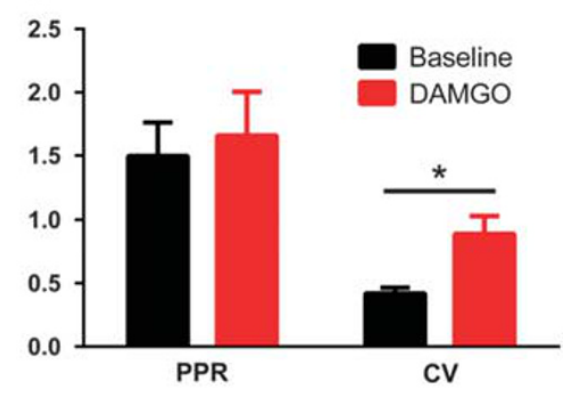

Figure I Mu opioid receptor (MOR) activation reduced evoked inhibitory postsynaptic current (elPSC) in the ventral periaqueductal gray/dorsal raphe (vPAG/DR) dopamine neurons. (a) Application of MOR agonist [D-Ala ${ }^{2}$, NMe-Phe ${ }^{4}$, Gly-ol ${ }^{5}$-enkephalin (DAMGO) reduced elPSC and was partially reversed by the antagonist D-Phe-Cys-Tyr-D-Trp-Orn-Thr-Pen-Thr-NH2 (CTOP). (b) DAMGO significantly increased the coefficient of variation (CV) but had no effect on paired-pulse ratio (PPR). * denotes $p<0.05$ in two-tailed paired Student's t-test.

\section{RESULTS}

\section{Mu Opioid Receptor Modulation of vPAG/DR TH Neurons}

Activation of MORs has been shown to inhibit GABAergic transmission onto VTA DA neurons in mice via a presynaptic mechanism, thus increasing the activity of DA neurons through disinhibition (Tan et al, 2010; Xie et al, 2012). In addition, MOR activation presynaptically reduces GABAergic transmission onto PAG neurons in rats (Vaughan et al, 1997; Hahm et al, 2011; Lecca et al, 2012). Thus, we initially sought to investigate the effects of MOR activation of GABAergic transmission onto vPAG/DR DA neurons by recording from TH-eGFP reporter mice (Li et al, 2013). We first examined the ability of the MOR agonist, DAMGO, to modulate electrically evoked IPSC (eIPSC) on vPAG/DR DA neurons. In addition to changes in current amplitude, we examined two parameters that are related to presynaptic release properties: paired pulse ratio (PPR), and coefficient of variation (CV). CV is defined as $\sigma / \mu$, where $\sigma$ is the standard deviation of IPSC amplitude and $\mu$ is the mean IPSC amplitude. At most central synapses, changes in PPR are associated with modifications of the release probability, while changes in $\mathrm{CV}$ are associated with alterations in the release probability or the number of release sites. We found that bath application of $300 \mathrm{nM}$ DAMGO led to a robust reduction of eIPSC amplitude ( $31.5 \pm 8.1 \%$ of baseline, $n=7$, $p=0.0002$; Figure 1a) that was partially reversed by application of the MOR antagonist CTOP $(62.4 \pm 10.6 \%$ of baseline, $p=0.01$ ). While PPR during DAMGO application $(1.66 \pm 0.4, n=7, p=0.44$; Figure $1 \mathrm{~b})$ did not differ from that of baseline $(1.50 \pm 0.3), \mathrm{CV}$ analysis revealed a significant change during DAMGO application $(0.89 \pm 0.1, n=7$, $p=0.02$; Figure $1 \mathrm{~b})$ compared with baseline $(0.42 \pm 0.05)$, suggestive of a presynaptic site of action. change in the number of release sites.

We next performed miniature IPSC recordings in the presence of TTX $(500 \mathrm{nM})$. Bath application of DAMGO $(300 \mathrm{nM})$ (representative traces; Figure 2a) for $10 \mathrm{~min}$ significantly decreased mIPSC frequency $(39.9 \pm 5.88 \%$ of baseline, $n=9, p=0.004$; Figure $2 \mathrm{~b}$ and $\mathrm{d}$ ) as well as amplitude $(85.88 \pm 4.11 \%$ of baseline, $n=9, p=0.0101$; Figure $2 \mathrm{c}$ and $\mathrm{e}$ ) in the vPAG/DR TH neurons. In a subset of these experiments, we explored the reversal of this inhibition (Figure 2d and e) and found that DAMGOinhibition of mIPSC frequency was reversed by CTOP $(95.8 \pm 12.0 \%$ of baseline, $n=7, \mathrm{~F}(1.366,8.197)=7.51$, $p=0.02)$ whereas amplitude was not $(72.66 \pm 6.5 \%$ of baseline, $\mathrm{F}(1.096,6.576)=1.593, p=0.25)$. This is consistent with the partial reversal of DAMGO effects on evoked IPSC shown earlier. To further confirm that the DAMGO effect observed was mediated through MOR activation, we next incubated the slices in CTOP for $>20$ min before and during DAMGO wash-on $(n=5)$. In the presence of the MOR antagonist CTOP, DAMGO application failed to attenuate mIPSC frequency $(99.2 \pm 2.9 \%$ of baseline; Figure $2 \mathrm{~h})$ or amplitude (97.2 $\pm 5.4 \%$ of baseline; Figure 2i). Finally, we examined the possibility of tonic MOR modulation on IPSC inputs to the vPAG/DR TH neurons. The application of CTOP alone did not affect mIPSC frequency $(109.7 \pm 4.8 \%$ of baseline, figure not shown) or amplitude $(96.4 \pm 9.0 \%$ of baseline), suggesting a lack of tonic GABA modulation by MOR in vPAG/DR TH neurons. Additionally, these CTOP antagonist data were collected as single 35 -min recording files ( 5 min baseline +20 min CTOP wash-on +10 min DAMGO application). The matching timeframe to DAMGO and CTOP reversal experiments provide support that the observed effects of DAMGO on mIPSCs were not due to rundown. Taken together, these results suggest that activation of MOR leads both to a reversible presynaptic inhibition as well as a more persistent postsynaptic inhibition of GABA function in vPAG/DR TH neurons.

\section{Anatomy of vPAG/DR TH Neurons}

We next explored the projections of vPAG/DR TH neurons using VTA TH neurons as an anatomical reference. In a TH-cre mouse line, we injected a cre-inducible virus expressing eYFP into the vPAG/DR (Figure 3a) and one expressing mCherry into the VTA. Serial sectioning of the brain revealed several target regions of the vPAG/DR DA neurons, of which the dBNST (Figure $3 \mathrm{~b}$ ) and the CeA (Figure 3c) received the densest projections. Of particular note, while the PAG projection to the rostral ventral lateral medulla (RVLM) has been suggested to play a role in the analgesic properties of opiates, we saw no evidence for a dopaminergic projection there (data not shown). This is 
consistent with a similar experiment (cre-inducible eYFP injected in to the PAG/DR of TH-cre mice) performed at the Allen Brain Institute (available online via the Allen Brain

a

BASELINE

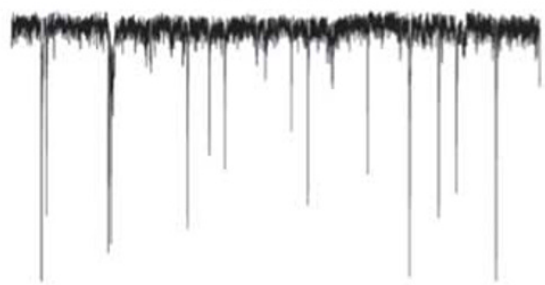

b
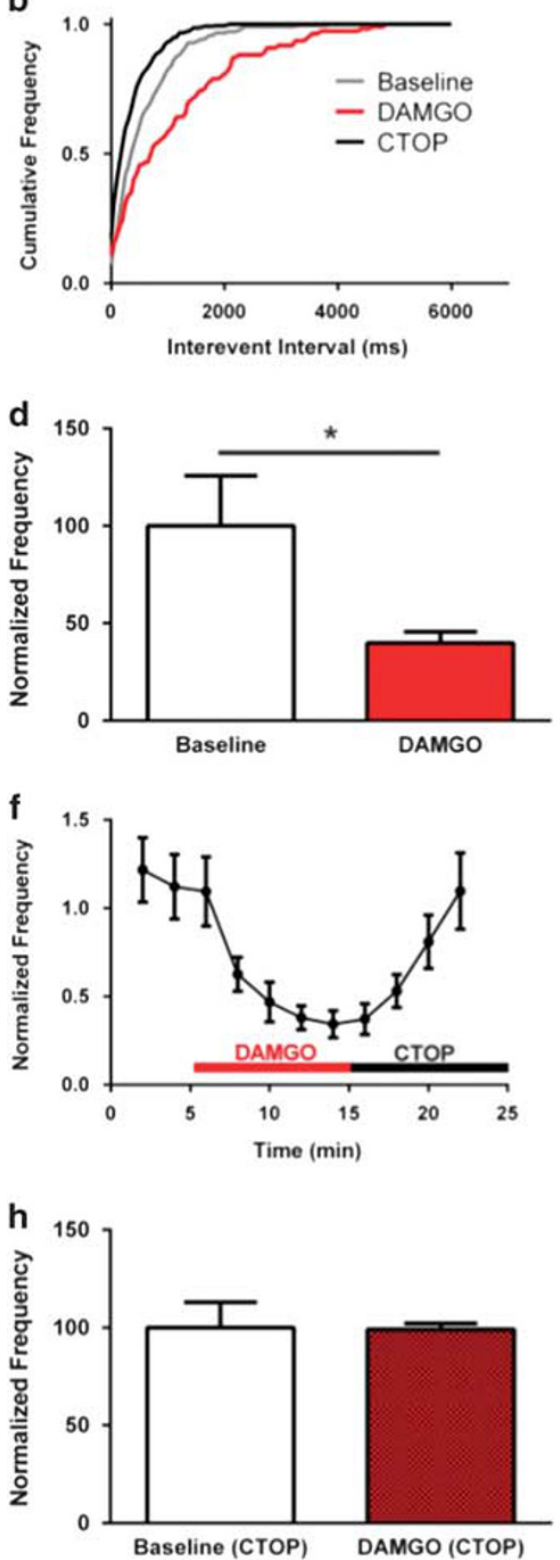

Connectivity Map) which found a near identical result. To more conclusively determine whether the vPAG/DR DA neurons project to the RVLM, we injected retrograde beads
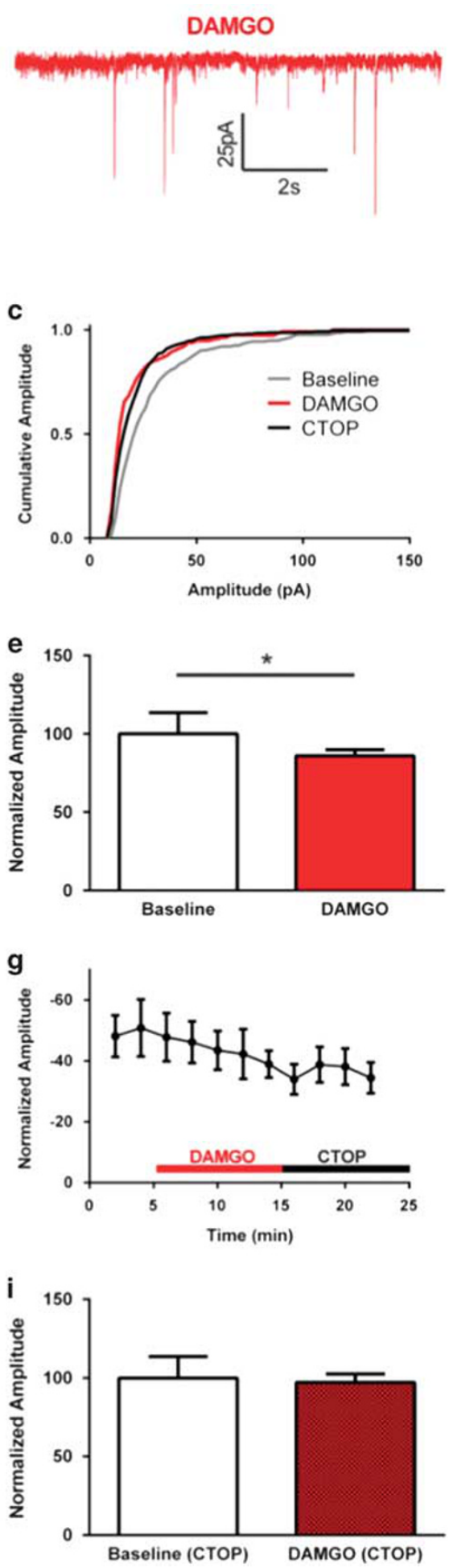

Figure 2 Mu opioid receptor (MOR) activation reduced mini inhibitory postsynaptic current (mIPSC) in the ventral periaqueductal gray/dorsal raphe (VPAG/DR) dopamine neurons. (a) Representative baseline and post-drug traces demonstrating the effect of $\left[\mathrm{D}-\mathrm{Ala}^{2}, \mathrm{NMe}-\mathrm{Phe}^{4}, \mathrm{Gly}_{\mathrm{o}} \mathrm{I}^{5}\right]$-enkephalin (DAMGO) on mIPSC in the VPAG/DR dopamine neurons. (b, c) Cumulative probability distribution of frequency (b) and peak amplitude (c) before application of DAMGO and followed by D-Phe-Cys-Tyr-D-Trp-Orn-Thr-Pen-Thr-NH 2 (CTOP). (d, e) Group effects $(n=10)$ demonstrating DAMGO significantly reduced both mIPSC frequency $(d)$ and amplitude (e). Time course demonstrating group $(n=7)$ DAMGO effects on frequency were reversed by the MOR antagonist CTOP (f); however, effects on amplitude were not reversed $(\mathrm{g})$. CTOP pretreatment blocked the DAMGO effects on mIPSC frequency (h) and amplitude (i) $(n=7)$. * denotes $p<0.05$ in two-tailed paired Student's $t$-test. 

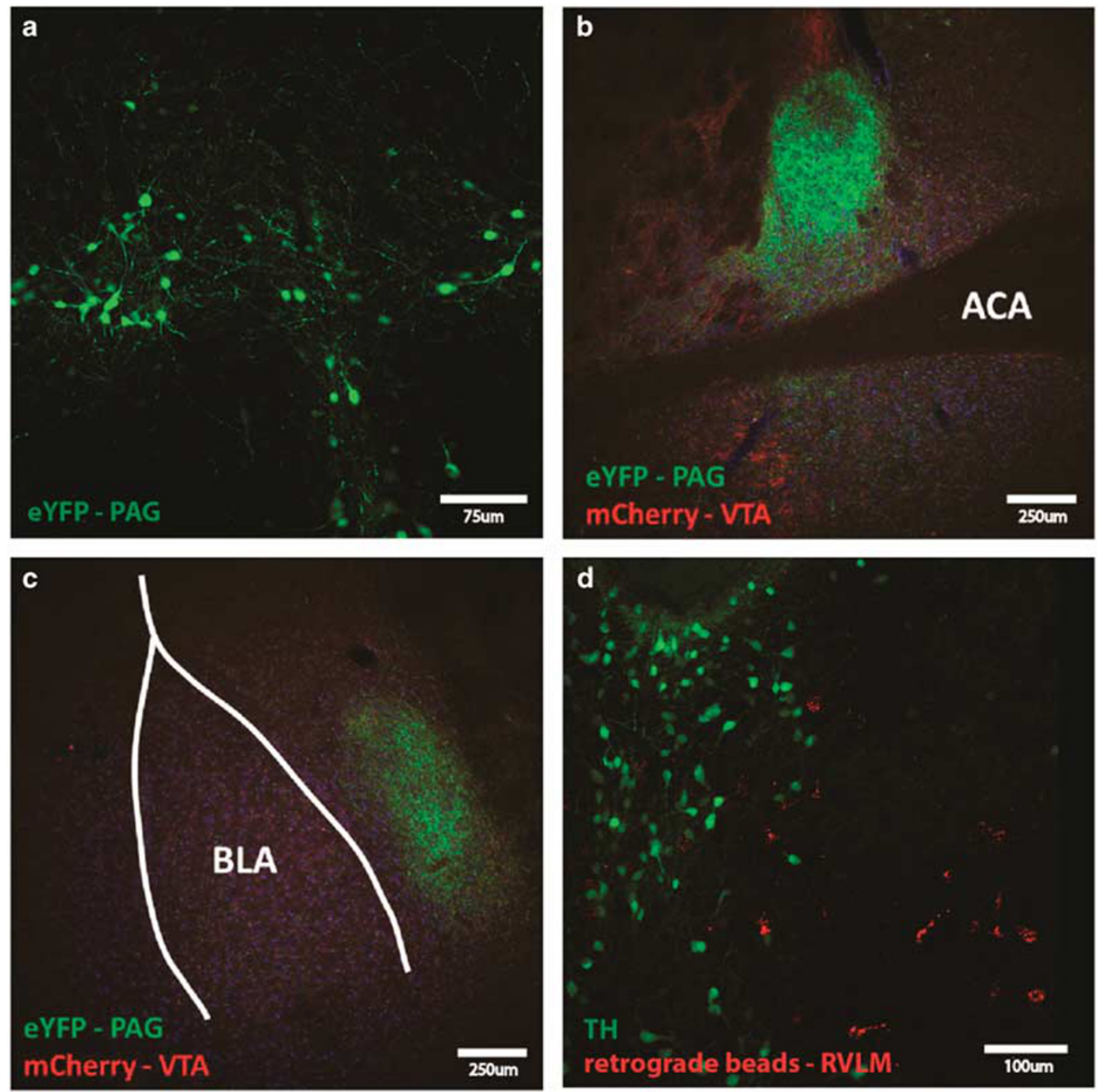

Figure 3 Ventral periaqueductal gray/dorsal raphe (VPAG/DR) tyrosine hydroxylase $(\mathrm{TH})$ neurons project to bed nucleus of the stria terminalis (BNST) and central nucleus of the amygdala (CeA), but not rostral ventral lateral medulla (RVLM). In a TH-cre mouse line, a cre-inducible enhanced yellow fluorescent protein (eYFP) viral construct was injected into the vPAG/DR (a), and a cre-inducible mCherry virus into the VTA. eYFP-expressing vPAG/DR TH terminals were observed in the BNST (b) and the CeA (c). In addition, when a retrograde bead injection was infused in the RVLM, neurons in the vPAG/DR that project to RVLM did not co-localize with TH (d).

into the RVLM in a TH-eGFP mouse. We found neural cell bodies with retrograde beads in the vPAG; however, they did not co-localize with TH-expressing neurons (Figure 3d). These results show that vPAG/DR DA neurons project to the extended amygdala, but not to the RVLM.

\section{Functional Neurochemistry of PAG/DR TH Neurons}

Midbrain DA neurons in the VTA and substantia nigra can co-release other neurotransmitters such as GABA (Tritsch et al, 2012) or glutamate (Hnasko et al, 2010; Stuber et al, 2010; Tecuapetla et al, 2010), yet this has not been assessed for PAG/DR TH neurons. We used immunohistochemistry in mouse reporter lines to determine co-localization of $\mathrm{TH}$ (Figure 4a) with markers of glutamate (vGlut2 reporter mice; Figure $4 \mathrm{~b}$ ) and GABA neurons (glutamate acid decarboxylase GAD67-GFP reporter mice; Figure 4d). Confocal images indicate that vPAG/DR TH-expressing neurons co-localize with vGlut2, but not GAD67 (Figure 4c and d).
Therefore, similar to DA neurons in the VTA, vPAG/DR DA neurons may co-release glutamate in downstream target regions.

We next investigated the possibility of DA/glutamate co-release using cre-inducible channelrhodopsin (ChR2eYFP) in the vPAG/DR of TH-cre mice. We first verified co-localization of ChR2-eYFP expression and TH-expressing neurons in the vPAG/DR (Figure 5a). Using cell-attached electrophysiology in the vPAG/DR ChR2-expressing cell bodies, action potentials were successfully triggered in a time-locked fashion with the presentation of blue LED at 1 , 5,10 , and $20 \mathrm{~Hz}$ (Figure 5b), demonstrating our capability to optically activate vPAG/DR TH neurons. Subsequently, we recorded optically evoked currents from neurons in the dBNST where vPAG/DR TH projections expressed ChR2. For these recordings, a Cs-methanesulfonate-based internal solution was used so that IPSCs were recorded at a holding potential of $+10 \mathrm{mV}$ (Figure 5c) and EPSCs were recorded at $-55 \mathrm{mV}$ (Figure $5 \mathrm{~d}$ ) within the same dBNST neuron. Of 

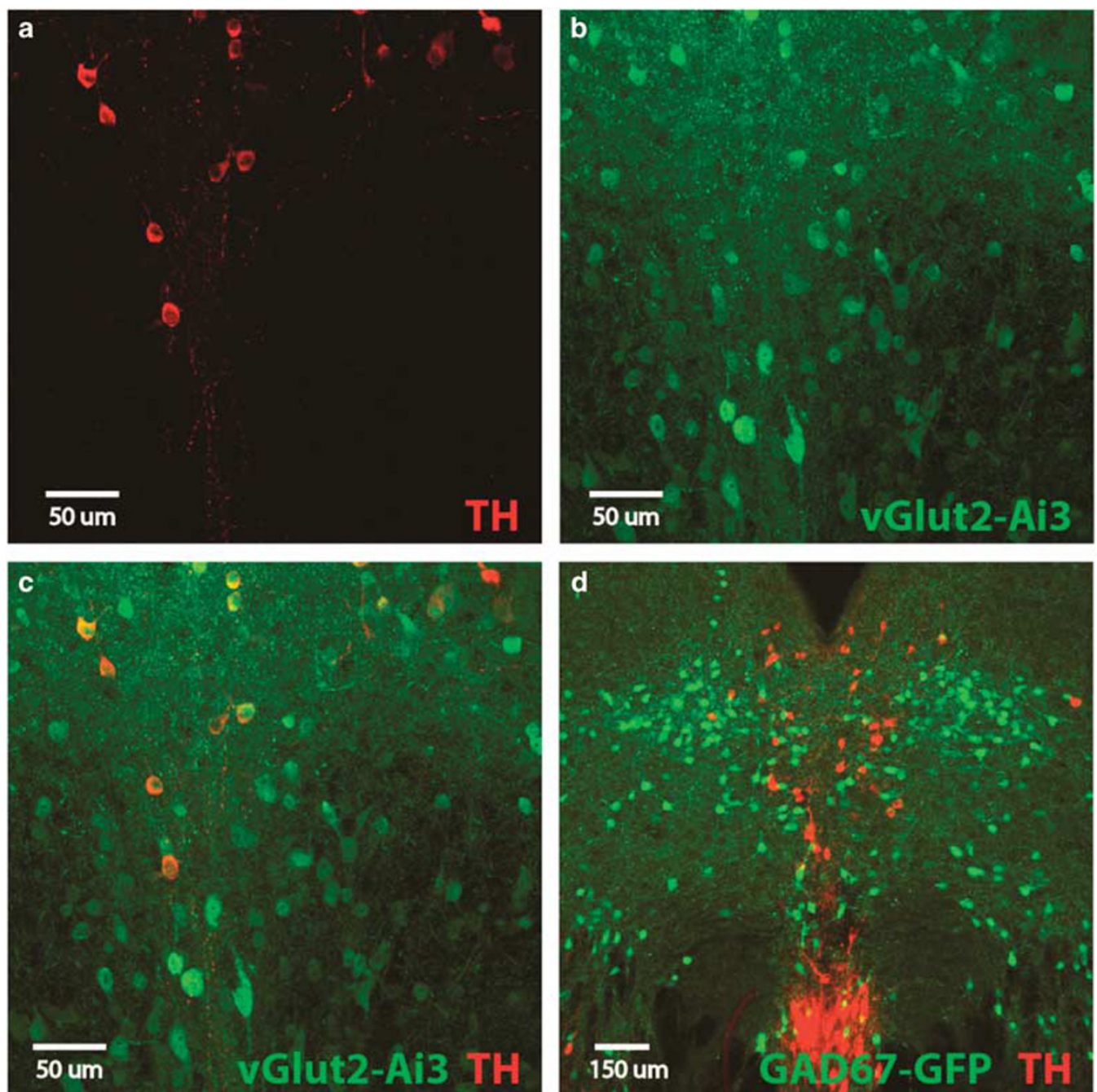

Figure 4 Ventral periaqueductal gray/dorsal raphe (vPAG/DR) tyrosine hydroxylase (TH)-positive neurons co-express vesicular glutamate type 2 transporter (vGlut2), but not markers for GABA transmission. (a-c) TH immunolabeling (A) in a vGlut2-Ai3 reporter mouse (b) demonstrated co-localization of a subpopulation of vPAG/DR TH expression and vGlut2 expression (c). (d) TH immunolabeling in a GAD67-GFP reporter mouse, illustrating vPAG/DR TH neurons and GABA neurons are distinct populations.

the 19 dBNST neurons recorded, 13 had only light-evoked glutamatergic currents, 4 had only light-evoked GABAergic currents, and 2 had both light-evoked glutamatergic and GABAergic currents. Upon inspecting the light stimulation to current delay, we found a significantly shorter delay of the glutamatergic currents $(2.9 \pm 0.16 \mathrm{~ms}$; Figure $5 \mathrm{e})$ relative to the GABAergic currents $(9.0 \pm 1.5 \mathrm{~ms}$. Student's $t$-test $p=0.007)$. The longer stimulation to current delay for GABAergic currents, as wells as the infrequent occurrence of light-evoked GABAergic currents, suggests that these inhibitory currents could be due to polysynaptic activation of GABA release, similar to what is seen in glutamatergic inputs from the basolateral amygdala (Kim et al, 2013b).

Having established that vPAG/DR TH neurons could release glutamate in the dBNST, we next utilized FSCV to examine if activation of vPAG/DR TH terminals could elicit DA release. In BNST slices from $\mathrm{TH}$-cre mice expressing ChR2-eYFP in vPAG/DR TH neurons, a CFME was placed in the dBNST where fluorescent expression of ChR2-eYFP was the strongest, and a blue LED was used to stimulate the slice. We observed time-locked release of DA (DA) upon optical stimulation of the slice. The current generated by DA release positively scaled with pulse number (Figure $5 \mathrm{f}$ ) and exhibited both a CV (Figure 5g) and a color-plot (Figure 5h) consistent with the oxidation and reduction of DA $(n=6)$. While CVs for DA and norepinephrine are similar, the enzyme necessary for norepinephrine synthesis, DA betahydroxylase, is not present in the vPAG region (Flores et al, 2004), thus our signal is likely DA. Together, these results suggest that in addition to co-expressing $\mathrm{TH}$ and vGlut2, vPAG/DR TH neurons co-release DA and glutamate in downstream target regions.

\section{Causal Assessment of PAG/DR TH Neurons in Pain-Related Behavior}

We next examined the behavioral consequences of vPAG/DR TH neuron activation using a chemogenetic approach. To 


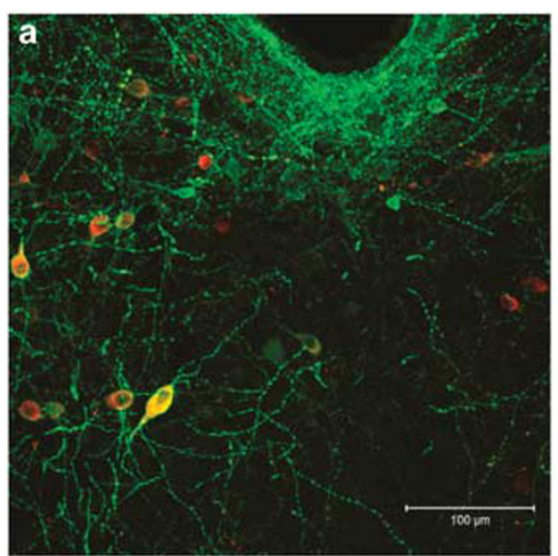

b
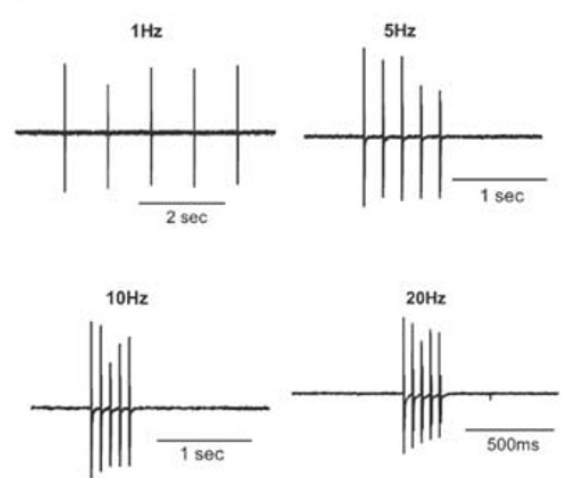

g
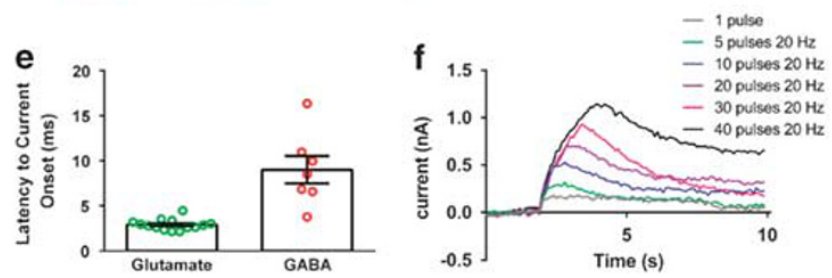

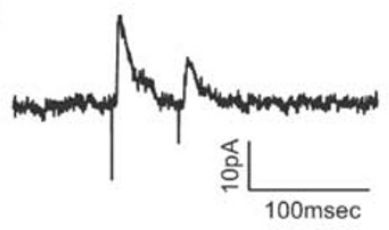

d
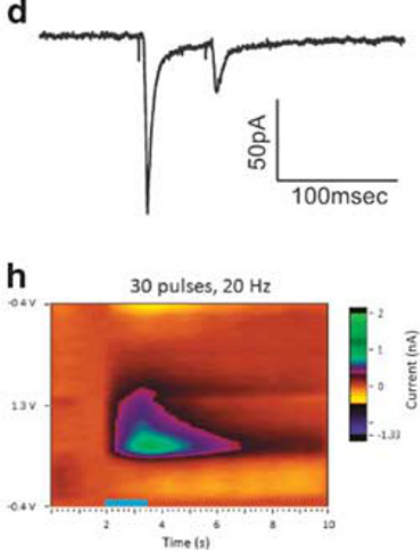

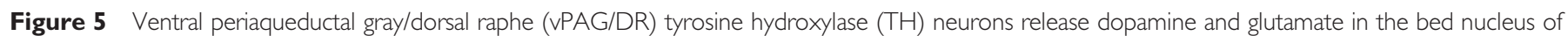

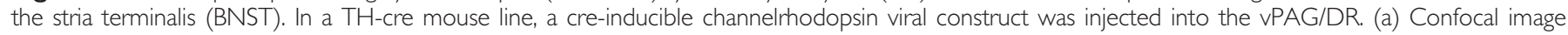

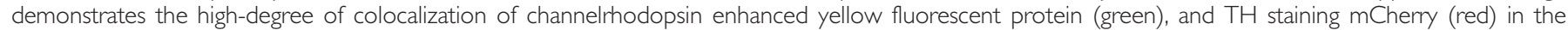

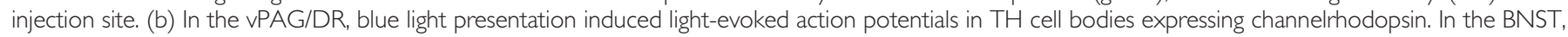

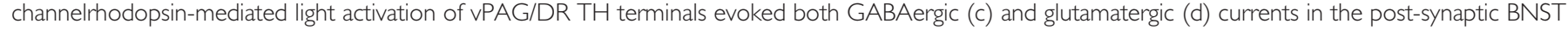

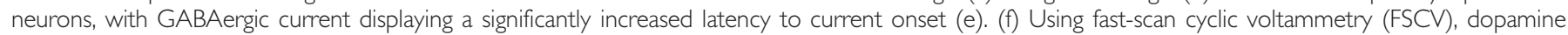

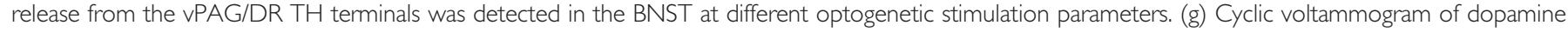
release. (h) FSCV color plot representing dopamine release.

selectively activate the vPAG/DR TH neurons, we used a cre-inducible viral construct to express an excitatory Gq-mediated DREADD in the vPAG/DR. Figure 6a shows the co-localization of the Gq DREADD with $\mathrm{TH}$ staining, confirming selective transduction in this TH-cre mouse line. We also examined overlap with serotonin neurons by co-staining with $\mathrm{TPH} 2$, a marker of 5HT neurons, and found no evidence of co-localization (data not shown). To functionally confirm the utility of the DREADD receptors in acute slice electrophysiology, we bath applied the synthetic DREADD ligand, clozapine- $N$-oxide (CNO, $10 \mu \mathrm{M})$, and observed significant membrane potential depolarization $(4.8 \pm 1.6 \mathrm{mV}, n=4$; Figure $6 \mathrm{~b})$ in DREADD-expressing neurons compared with the control mCherry-expressing neurons. Having validated this approach ex vivo, we next probed the role of vPAG/DR DA neurons in two different nociception assays: the hot plate assay and the tail flick assay. The tail flick response is a reflex response that can be elicited after spinal cord transection (Franklin and Abbott, 1989); however, the hot plate assay measures behavioral outputs such as jumping, hindpaw withdrawal, and hindpaw licking that requires supraspinal modulation, rather than reflexes. In the hot plate assay, Gq DREADD activation via intraperitoneal (i.p.) $1 \mathrm{mg} / \mathrm{kg} \mathrm{CNO}$ administration significantly increased latency to paw withdrawal $(46.1 \pm 1.8 \mathrm{~s}, n=10 \mathrm{Gq}$ DREADD and 10 Control, $p=0.03$; Figure $6 \mathrm{c}$ ) in comparison to controls $(38.7 \pm 2.7 \mathrm{~s})$, suggesting an antinociceptive effect upon vPAG/DR TH neuron activation. vPAG/DR TH activation, however, showed no effect in the tail flick assay (Figure 6d). In order to verify that the increase in hot plate paw withdrawal latency was not due to changes in locomotor function, we measured distance traveled in an open field assay and observed no differences in either the Gq DREADD or control conditions (Figure 6e).

\section{DISCUSSION}

The results of this study provide evidence that MOR activation inhibits GABAergic inputs onto vPAG/DR DA neurons through both presynaptic and postsynatpic mechanisms of action. Moreover, we found that activation of vPAG/DR TH neurons can modulate a subclass of pain-related behaviors, and that these neurons overlap with a population of vGlut2 neurons to functionally release both DA and glutamate in the BNST. Together, this suggests that the DA neurons of the vPAG/DR are a critical neuronal substrate for opiate-induced regulation of pain.

\section{MOR Actions on vPAG/DR DA Neurons}

Similar to studies on DA neurons in the VTA, we found that ex vivo bath application of the MOR ligand DAMGO significantly reduced evoked IPSCs as well as mIPSC frequency and amplitude. In the evoked experiments, the inhibiting effect of DAMGO was partially reversible by application of the antagonist, while in the miniature experiments, the effect of DAMGO on frequency was completely reversed by the antagonist, but not the amplitude. Overall, this is consistent with a model in which there is 

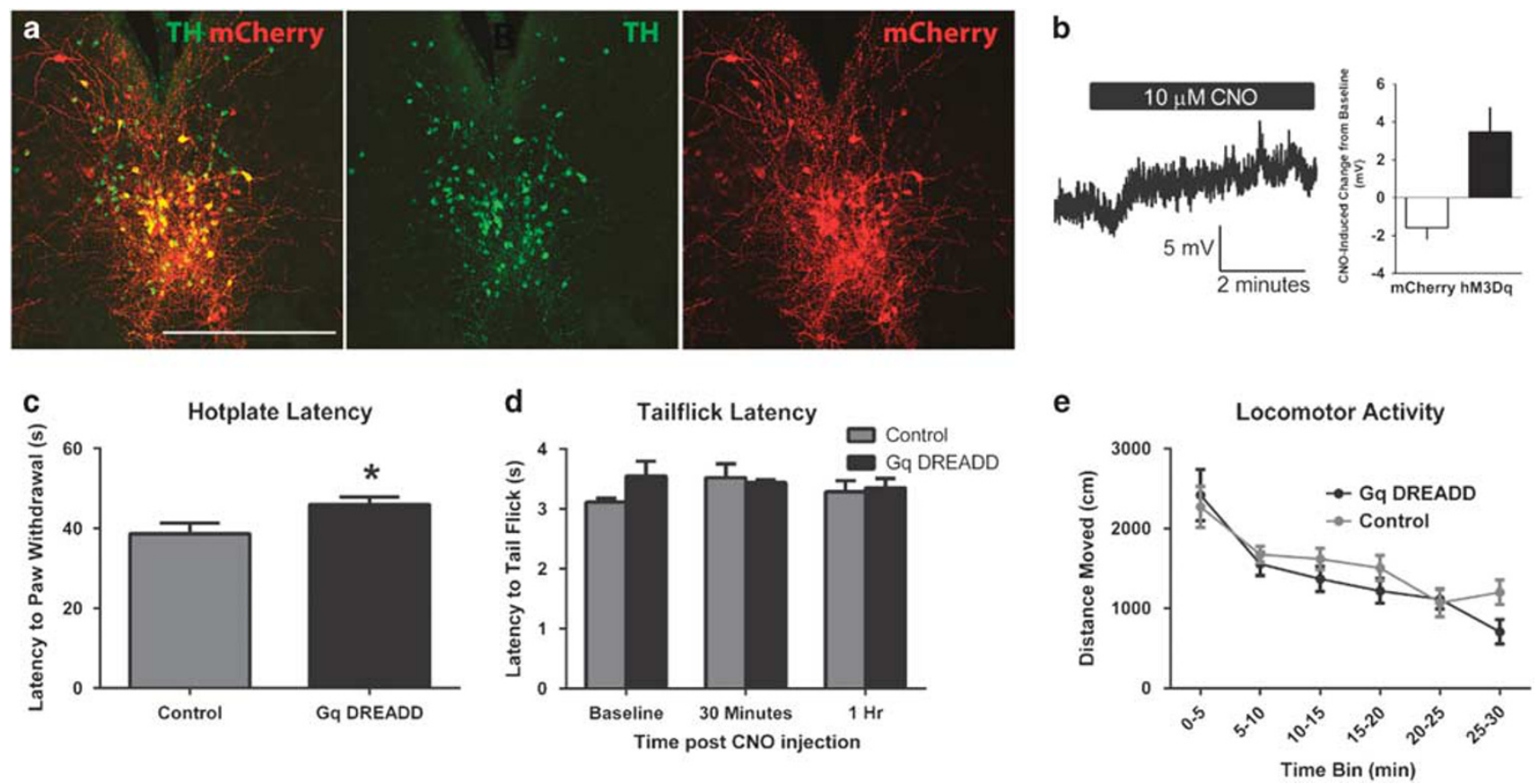

Figure 6 Activation of Gq signaling in ventral periaqueductal gray/dorsal raphe (vPAG/DR) tyrosine hydroxylase (TH) neurons reduces response in hotplate but not tail flick or locomotor activity. Gq designer receptors exclusively activated by designer drug (DREADD) was virally expressed in vPAG/DR DA neurons in a TH-cre mouse. (a) Confocal images demonstrated colocalization of Gq DREADD-mCherry, and TH staining GFP in the injection site. (b) In slice electrophysiology, bath application of CNO $(10 \mu \mathrm{M})$ depolarized vPAG/DR TH neurons. Chemogenetic activation of vPAG/DR TH neurons significantly increased latency to paw withdrawal in hotplate assay (c). However, no effects were observed in tail flick latency (d) or locomotion (e). * denotes $p<0.05$ in two-tailed paired Student's t-test.

acute suppression of GABA release following activation of MOR, similar to other studies (Johnson and North, 1992; Lupica, 1995; Bergevin et al, 2002). However, our results also suggest a potential persistent postsynaptic inhibition, which to date has not been reported. This is particularly intriguing in light of recent evidence for postsynaptic MOR function in VTA DA neurons (Margolis et al, 2014). These results suggest that MOR activation reduces inhibitory input and therefore potentially disinhibits $\mathrm{vPAG} / \mathrm{DR}$ DA neurons in order to modulate downstream circuits.

\section{Functional Neuroanatomy of vPAG/DR DA Neurons}

We used a viral-based tracing approach to examine the outputs of vPAG/DR DA neurons. Consistent with several other studies, we found that these neurons project densely to the extended amygdala; in particular, the BNST and the CeA (Rizvi et al, 1991; Vianna and Brandao, 2003; Kim et al, 2013a). Interestingly, while VTA DA neurons have similar outputs, there appeared to be distinct patterns of innervations when we directly compared the projections. The vPAG/ DR DA neurons appeared to strongly innervate the oval nucleus of the BNST, whereas VTA DA neurons target a portion of the BNST lateral to the oval nucleus. These distinct projections suggest that activation of these inputs may lead to recruitment of different microcircuits, an important possibility, given the heterogeneity of the BNST (Kim et al, 2013b). Interestingly, we found no evidence for innervation of the RVLM by vPAG/DR DA neurons using either viral-based mapping or classic retrograde labeling. Given that local DA signaling in the PAG/DR can modulate pain-related behaviors, this suggests that vPAG/DR DA neurons may serve to locally modulate RVLM-projecting neurons in addition to modulation of distinct target regions, which may be important for coordinating the emotional and visceral aspects of pain.

Following the examination of projection targets, we used immunohistochemistry in well-characterized reporter mice to demonstrate that vPAG/DR DA neurons co-express vesicular glutamate transporter 2 (vGlut2), but are not GABAergic. Using a ChR2eYFP-based mapping approach, we then functionally assessed neurotransmitter release in the $\mathrm{BNST}$, a region that receives dense innervation of $\mathrm{vPAG} / \mathrm{DR}$ DA neurons. We found via cyclic voltammetry, for the first time, that stimulation of vPAG/DR DA neurons could release both DA and glutamate in the BNST. Interestingly, the vast majority of cells recorded received glutamatergic input (68\%) upon optical stimulation while few cells $(21 \%)$ received inhibitory or mixed (11\%) excitatory/inhibitory input. This was surprising given our immunohistochemistry data showing that vPAG/DR DA neurons co-express vGlut2 but not GAD67. The light-evoked GABAergic currents, however, exhibit a significantly longer stimulation-to-current onset delay when compared with the glutamatergic currents. These GABAergic currents are likely due to a local polysynaptic microcircuit engaged by $\mathrm{DA}$ or glutamate release from the vPAG/DR DA rgic projections, a model consistent with results demonstrating that DA can depolarize a discrete subpopulation of neurons in the BNST (Kash et al, 2008; Silberman and Winder, 2013). However, it is important to recognize one potential limitation of our findings regarding co-release of DA and glutamate. While our 
staining revealed that ChR2-eYFP was expressed predominantly in TH-expressing neurons, there were a small number of neurons that did not appear to express detectable levels of $\mathrm{TH}$ protein. It is possible that some of these neurons express cre under the TH promoter but do not express $\mathrm{TH}$ protein, resulting in non-dopaminergic glutamate neurons expressing ChR2-eYFP in our preparation, and resulting in glutamate release upon light activation. This possibility, however, is mitigated by our staining demonstrating co-expression of $\mathrm{TH}$ in vGlut2-positive neurons.

Our results demonstrate the release of both glutamate and DA in the BNST from vPAG DA neurons. In addition to the substantial modulatory role of the vPAG/DR in nociception, the BNST also demonstrates pain-dependent neural responses in rats (Morano et al, 2008). Lesion of the BNST blocks pain-induced conditioned place aversion (Deyama et al, 2007), and prevents shock-induced sensitization of the vocalization response to heat (Crown et al, 2000). Furthermore, the BNST modulates the emotional aspect of pain (Minami and Ide, 2015). These data, in conjunction with our data, suggest that input from the vPAG/DR DA neurons modulate BNST functions pertaining to pain.

\section{Chemogenetic Activation of vPAG/DR Neurons Revealed a Role in Pain-Related Behaviors}

Lastly, via chemogenetic techniques using the excitatory Gq DREADDs, we probed the functional consequences of selective vPAG/DR DA neuron activation in two different nociception assays: the hot plate and the tail flick tests to assess changes in supraspinal and reflex response, respectively. Previous studies have suggested that vPAG/DR activation mediates antinociception in a supraspinal manner, consistent with our findings that activation of vPAG/DR DA neurons increased latency to hindpaw withdrawal in the hot plate assay, but did not alter responses in the tail flick assay (Flores et al, 2004). It is unlikely that this difference was due to altered locomotion following activation of these neurons, as we detected no significant difference in movement between Gq DREADD activation of vPAG/DR DA neurons compared with mCherry controls.

\section{CONCLUSION}

Here we provide the first demonstration that the MOR inhibits GABA function in $\mathrm{vPAG} / \mathrm{DR}$ DA neurons, primarily through inhibition of release. Moreover, we demonstrate that these neurons densely innervate the extended amygdala (specifically the BNST) where they can release both glutamate and DA. Finally, we demonstrate that chemogenetic activation of vPAG/DR DA neurons can alter painrelated behaviors. Taken together, this study provides a mechanistic understanding of how MOR activation within the vPAG/DR can alter nociception, and provides a discrete cellular target for development of novel pain medications.

\section{FUNDING AND DISCLOSURE}

The authors declare no conflict of interest. This work was funded by grants from the NIH, the Brain and Behavior Research Foundation and the Bowles Center for Alcohol Studies.

\section{REFERENCES}

Akaike A, Shibata T, Satoh M, Takagi H (1978). Analgesia induced by microinjection of morphine into, and electrical stimulation of, the nucleus reticularis paragigantocellularis of rat medulla oblongata. Neuropharmacology 17: 775-778.

Bath BD, Michael DJ, Trafton BJ, Joseph JD, Runnels PL, Wightman RM (2000). Subsecond adsorption and desorption of dopamine at carbon-fiber microelectrodes. Anal Chem 72: 5994-6002.

Beiske AG, Loge JH, Ronningen A, Svensson E (2009). Pain in Parkinson's disease: prevalence and characteristics. Pain 141: 173-177.

Bergevin A, Girardot D, Bourque MJ, Trudeau LE (2002). Presynaptic mu-opioid receptors regulate a late step of the secretory process in rat ventral tegmental area GABAergic neurons. Neuropharmacology 42: 1065-1078.

Budai D, Fields HL (1998). Endogenous opioid peptides acting at mu-opioid receptors in the dorsal horn contribute to midbrain modulation of spinal nociceptive neurons. J Neurophysiol 79: 677-687.

Crown ED, King TE, Meagher MW, Grau JW (2000). Shockinduced hyperalgesia: III. Role of the bed nucleus of the stria terminalis and amygdaloid nuclei. Behav Neurosci 114: 561-573.

Deyama S, Nakagawa T, Kaneko S, Uehara T, Minami M (2007). Involvement of the bed nucleus of the stria terminalis in the negative affective component of visceral and somatic pain in rats. Behav Brain Res 176: 367-371.

Dougalis AG, Matthews GA, Bishop MW, Brischoux F, Kobayashi K, Ungless MA (2012). Functional properties of dopamine neurons and co-expression of vasoactive intestinal polypeptide in the dorsal raphe nucleus and ventro-lateral periaqueductal grey. Eur J Neurosci 36: 3322-3332.

Espejo EF, Stinus L, Cador M, Mir D (1994). Effects of morphine and naloxone on behaviour in the hot plate test: an ethopharmacological study in the rat. Psychopharmacology 113: 500-510.

Finn DP, Jhaveri MD, Beckett SR, Roe CH, Kendall DA, Marsden CA et al (2003). Effects of direct periaqueductal grey administration of a cannabinoid receptor agonist on nociceptive and aversive responses in rats. Neuropharmacology 45: 594-604.

Flores JA, El Banoua F, Galan-Rodriguez B, Fernandez-Espejo E (2004). Opiate anti-nociception is attenuated following lesion of large dopamine neurons of the periaqueductal grey: critical role for D1 (not D2) dopamine receptors. Pain 110: 205-214.

Franklin KJ, Abbott F (1989). Techniques for assessing the effects of drugs on nociceptive responses. In: Boulton A et al (ed). Psychopharmacology, Vol. 13, Humana Press: Clifton, NJ, pp 145-216.

Hahm ET, Kim Y, Lee JJ, Cho YW (2011). GABAergic synaptic response and its opioidergic modulation in periaqueductal gray neurons of rats with neuropathic pain. BMC Neurosci 12: 41.

Heimer R, Zhan W, Grau LE (2015). Prevalence and experience of chronic pain in suburban drug injectors. Drug Alcohol Depend 151: $92-100$.

Hnasko TS, Chuhma N, Zhang H, Goh GY, Sulzer D, Palmiter RD et al (2010). Vesicular glutamate transport promotes dopamine storage and glutamate corelease in vivo. Neuron 65: 643-656.

Hong J, Novick D, Montgomery W, Aguado J, Duenas H, Peng X et al (2015). Should unexplained painful physical symptoms be considered within the spectrum of depressive symptoms? Clin Pract Epidemiol Ment Health 11: 130-139.

Jarcho JM, Mayer EA, Jiang ZK, Feier NA, London ED (2012). Pain, affective symptoms, and cognitive deficits in patients with cerebral dopamine dysfunction. Pain 153: 744-754.

Johnson SW, North RA (1992). Opioids excite dopamine neurons by hyperpolarization of local interneurons. J Neurosci 12: 483-488. 
Kash TL, Nobis WP, Matthews RT, Winder DG (2008). Dopamine enhances fast excitatory synaptic transmission in the extended amygdala by a CRF-R1-dependent process. J Neurosci 28: $13856-13865$.

Kim EJ, Horovitz O, Pellman BA, Tan LM, Li Q, Richter-Levin G et al (2013a). Dorsal periaqueductal gray-amygdala pathway conveys both innate and learned fear responses in rats. Proc Natl Acad Sci USA 110: 14795-14800.

Kim SY, Adhikari A, Lee SY, Marshel JH, Kim CK, Mallory CS et al (2013b). Diverging neural pathways assemble a behavioural state from separable features in anxiety. Nature 496: 219-223.

Lecca S, Melis M, Luchicchi A, Muntoni AL, Pistis M (2012). Inhibitory inputs from rostromedial tegmental neurons regulate spontaneous activity of midbrain dopamine cells and their responses to drugs of abuse. Neuropsychopharmacology 37: 1164-1176.

Li C, McCall NM, Lopez AJ, Kash TL (2013). Alcohol effects on synaptic transmission in periaqueductal gray dopamine neurons. Alcohol 47: 279-287.

Lupica CR (1995). Delta and mu enkephalins inhibit spontaneous GABA-mediated IPSCs via a cyclic AMP-independent mechanism in the rat hippocampus. J Neurosci 15: 737-749.

Margolis EB, Hjelmstad GO, Fujita W, Fields HL (2014). Direct bidirectional mu-opioid control of midbrain dopamine neurons. J Neurosci 34: 14707-14716.

Mayer DJ, Wolfle TL, Akil H, Carder B, Liebeskind JC (1971). Analgesia from electrical stimulation in the brainstem of the rat. Science 174: 1351-1354.

Minami M, Ide S (2015). How does pain induce negative emotion? Role of the bed nucleus of the stria terminalis in pain-induced place aversion. Curr Mol Med 15: 184-190.

Morano TJ, Bailey NJ, Cahill CM, Dumont EC (2008). Nuclei-and condition-specific responses to pain in the bed nucleus of the stria terminalis. Prog Neuropsychopharmacol Biol Psychiatry 32: 643-650.

Rizvi TA, Ennis M, Behbehani MM, Shipley MT (1991). Connections between the central nucleus of the amygdala and the midbrain periaqueductal gray: topography and reciprocity. J Comp Neurol 303: 121-131.

Silberman Y, Winder DG (2013). Corticotropin releasing factor and catecholamines enhance glutamatergic neurotransmission in the lateral subdivision of the central amygdala. Neuropharmacology 70: 316-323.

Stuber GD, Hnasko TS, Britt JP, Edwards RH, Bonci A (2010). Dopaminergic terminals in the nucleus accumbens but not the dorsal striatum corelease glutamate. J Neurosci 30: 8229-8233.

Tan KR, Brown M, Labouebe G, Yvon C, Creton C, Fritschy JM et al (2010). Neural bases for addictive properties of benzodiazepines. Nature 463: 769-774.

Tecuapetla F, Patel JC, Xenias H, English D, Tadros I, Shah F et al (2010). Glutamatergic signaling by mesolimbic dopamine neurons in the nucleus accumbens. J Neurosci 30: 7105-7110.

Tritsch NX, Ding JB, Sabatini BL (2012). Dopaminergic neurons inhibit striatal output through non-canonical release of GABA. Nature 490: 262-266.

Tykocki T, Kornakiewicz A, Mandat T, Nauman P (2013). Pain perception in patients with Parkinson's disease. J Clin Neurosci 20: 663-666.

Vaughan CW, Ingram SL, Connor MA, Christie MJ (1997). How opioids inhibit GABA-mediated neurotransmission. Nature 390: 611-614.

Vianna DM, Brandao ML (2003). Anatomical connections of the periaqueductal gray: specific neural substrates for different kinds of fear. Braz J Med Biol Res 36: 557-566.

Weaver M, Schnoll S (2002). Abuse liability in opioid therapy for pain treatment in patients with an addiction history. Clin J Pain 18: S61-S69.

Wood PB (2008). Role of central dopamine in pain and analgesia. Expert Rev Neurother 8: 781-797.

Xie G, Hipolito L, Zuo W, Polache A, Granero L, Krnjevic K et al (2012). Salsolinol stimulates dopamine neurons in slices of posterior ventral tegmental area indirectly by activating mu-opioid receptors. J Pharmacol Exp Ther 341: 43-50. 